\title{
Análisis y evaluación del riesgo de deslizamientos superficiales en un terreno montañoso tropical: implementación de modelos físicos simples
}

\author{
Shallow landslides risk assessment in a tropical mountain terrain: implementation \\ of simple physical models
}

\author{
R.J. Marín ; J. Marín-Londoño ; Á.J. Mattos iD
}

\begin{abstract}
Shallow landslide risk denotes the losses or damages caused by the occurrence of this phenomenon and depends on the magnitude, related to its probability of occurrence, and its effect on the exposed elements, determined by its degree of vulnerability. In this research, a hazard, vulnerability and risk assessment of shallow landslides in a tropical mountainous terrain is carried out. The physical model SHALSTAB was used for the hazard assessment and other models of easy implementation to calculate the physical vulnerability of 8 dwellings and of the people within them. The risk of the structures is calculated, in economic terms, and the risk of loss of human lives, for people inside the dwellings, by means of an analysis of the consequences of these exposed elements given the occurrence of shallow landslides defined in the evaluation of the hazard. This methodology represents a tool that can be useful for land use planning and landslide risk management.
\end{abstract}

Index Terms - Hazard, risk, shallow landslides, SHALSTAB, T-model, vulnerability.

Resumen - El riesgo de deslizamientos superficiales expresa las pérdidas o daños causados por la ocurrencia de este fenómeno y depende de la magnitud, relacionada con su probabilidad de ocurrencia, y su efecto en los elementos expuestos, determinado por su grado de vulnerabilidad. En esta investigación se analizan y evalúan la amenaza, vulnerabilidad y riesgo de deslizamientos superficiales en un terreno montañoso tropical. Se utilizó el modelo físico SHALSTAB para el análisis de la amenaza y otros modelos de fácil implementación para calcular la vulnerabilidad física de ocho viviendas y de personas dentro de las mismas. Se calcula el riesgo de las estructuras, en términos económicos, y el

Este manuscrito fue enviado el 01 de agosto de 2019 y aceptado el 16 de marzo de 2020. Los autores agradecen al Grupo de Investigación en Infraestructura (GII) y a la Universidad de Antioquia por el apoyo en el desarrollo de esta investigación.

R.J. Marín, Escuela Ambiental, Facultad de Ingeniería, Universidad de Antioquia UdeA. Calle 70 \#52-21, Medellín, Colombia (rjose.marin@udea.edu.co). riesgo de pérdidas de vidas humanas, para personas dentro de las viviendas, mediante un análisis de las consecuencias de estos elementos expuestos dada la ocurrencia de deslizamientos superficiales definidos en la evaluación de la amenaza. Esta metodología representa una herramienta que puede ser útil en planes de ordenamiento territorial y gestión del riesgo de movimientos en masa.

Palabras claves - Amenaza, deslizamientos superficiales, modelo $T$, riesgo, SHALSTAB, vulnerabilidad.

\section{INTRODUCCIÓN}

$\mathrm{E}$ $\mathrm{L}$ riesgo de movimientos en masa es una medida de la probabilidad y severidad de este fenómeno a la salud, bienes materiales o al medio ambiente. Suele calcularse como el producto entre la probabilidad de su ocurrencia (para una magnitud dada) y las consecuencias [1].

El análisis y la evaluación del riesgo de movimientos en masa se pueden llevar a cabo tanto a escala regional como de sitio específico. Estos son esenciales para desarrollar políticas apropiadas en la gestión del riesgo de desastres naturales y específicamente en la reducción de los daños causados por la ocurrencia de este fenómeno [2]. Si bien la información obtenida en un correcto análisis y evaluación de movimientos en masa podría contribuir en el desarrollo de estrategias de reducción de pérdidas, muy pocos estudios incluyen la preparación de mapas de riesgo [3].

Los mapas de riesgo de movimientos en masa se basan en los pronósticos o predicciones del análisis de amenaza (representado generalmente como otro mapa) y presentan las pérdidas financieras esperadas, o para un escenario de riesgo determinado, causadas por la ocurrencia de estos fenómenos [4]. Se pueden basar en un análisis determinístico,

J. Marín-Londoño, Escuela Ambiental, Facultad de Ingeniería, Universidad de Antioquia UdeA. Calle 70 \#52-21, Medellín, Colombia.

Á.J. Mattos, Escuela Ambiental, Facultad de Ingeniería, Universidad de Antioquia UdeA. Calle 70 \#52-21, Medellín, Colombia. 
probabilístico o la combinación de estos. Si bien el análisis y la evaluación del riesgo debería llevarse a cabo mediante un análisis cuantitativo, los resultados suelen simplificarse en términos cualitativos, como podrían ser unos mapas con niveles de amenaza de movimientos en masa baja, media y alta (de igual forma para vulnerabilidad y riesgo) [5]. En términos ingenieriles, esta representación cualitativa puede ser insuficiente para la toma de decisiones porque desestima datos importantes. Un experto en el tema debería tener las herramientas para interpretar los resultados cuantitativos en los cuales se basan los mapas de clasificación cualitativa de amenaza, vulnerabilidad o riesgo.

Una gran ventaja de los análisis de riesgo cuantitativos es que permiten calcular el riesgo de una forma objetiva y reproducible, de modo que los resultados pueden ser comparados con diferentes escenarios. De igual forma, permiten realizar un análisis de costo-beneficio y sirven como una base para decidir las acciones de manejo o mitigación [6].

Debido al rechazo de la sociedad a incidentes con muchas fatalidades, el concepto de riesgo social toma relevancia y se relaciona con el riesgo de múltiples pérdidas de vidas humanas o lesiones para la población. En este sentido, la sociedad llevaría la carga de un movimiento en masa que genera cierto número de muertes, heridos, pérdidas económicas, ambientales o de otro tipo. Este riesgo se puede medir en términos de la probabilidad acumulada anual de que un número de vidas se pierdan en un escenario determinado [1], [7].

En el Valle de Aburrá, como en el resto de Colombia, son escasas las investigaciones [8]-[10] que involucran el análisis del riesgo de movimientos en masa. Sin embargo, al ser una región montañosa ubicada en la zona intertropical, es alto el potencial de ocurrencia de este fenómeno. De hecho, los movimientos en masa inciden en el $77 \%$ de las fatalidades totales que se presentan por fenómenos de origen natural, sin incluir las avenidas torrenciales (13\%) [11].

Esta investigación presenta una metodología basada en modelos físicos de amenaza y vulnerabilidad de movimientos en masa para evaluar el riesgo de deslizamientos superficiales en un terreno montañoso tropical del Valle de Aburrá (Colombia).

\section{Metodología}

\section{A. Sitio de estudio}

En el área de estudio $\left(2.85 \mathrm{~km}^{2}\right)$ se tienen tres pequeñas subcuencas que incluyen un tramo vial de la Autopista Medellín-Bogotá, entre los kilómetros 12+350 y 15+440, en el Municipio de Copacabana (Antioquia, Colombia). El rango de elevaciones se encuentra entre 1751 m.s.n.m y 2549 m.s.n.m. La Fig. 1 muestra la localización del sitio de estudio.

En el área de interés se presenta el contacto geólogico de Anfibolitas de Medellín y Gneiss de La Ceja. Esta última unidad geológica subyace a depósitos de vertientes en la parte baja de la subcuenca que abarca una mayor área del sitio de estudio (Fig. 2a). Las Anfibolitas se componen de minerales de horblenda, plagioclasa y cuarzo, desarrollando un suelo residual de limo de color amarillo-rojizo. En el horizonte superficial

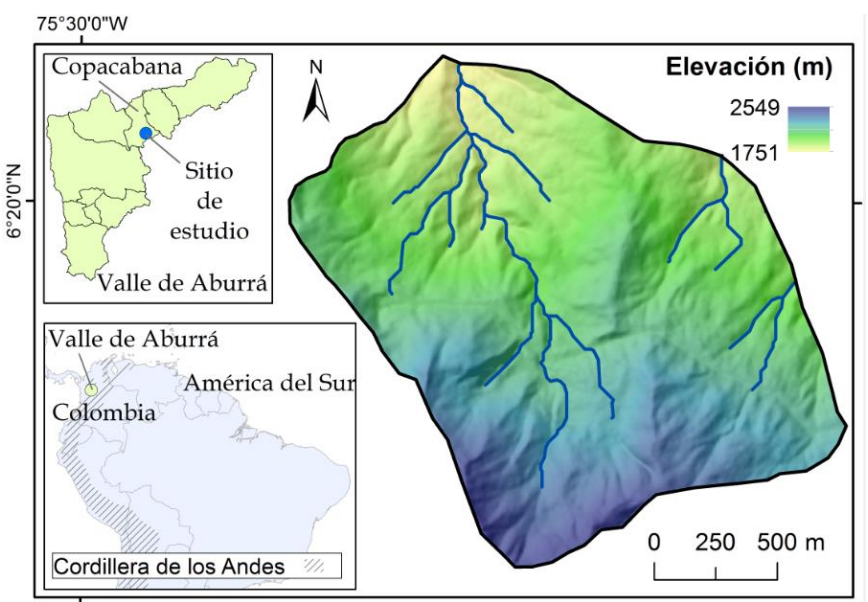

Fig. 1. Localización y modelo digital de elevación del sitio de estudio (Copacabana, Colombia).
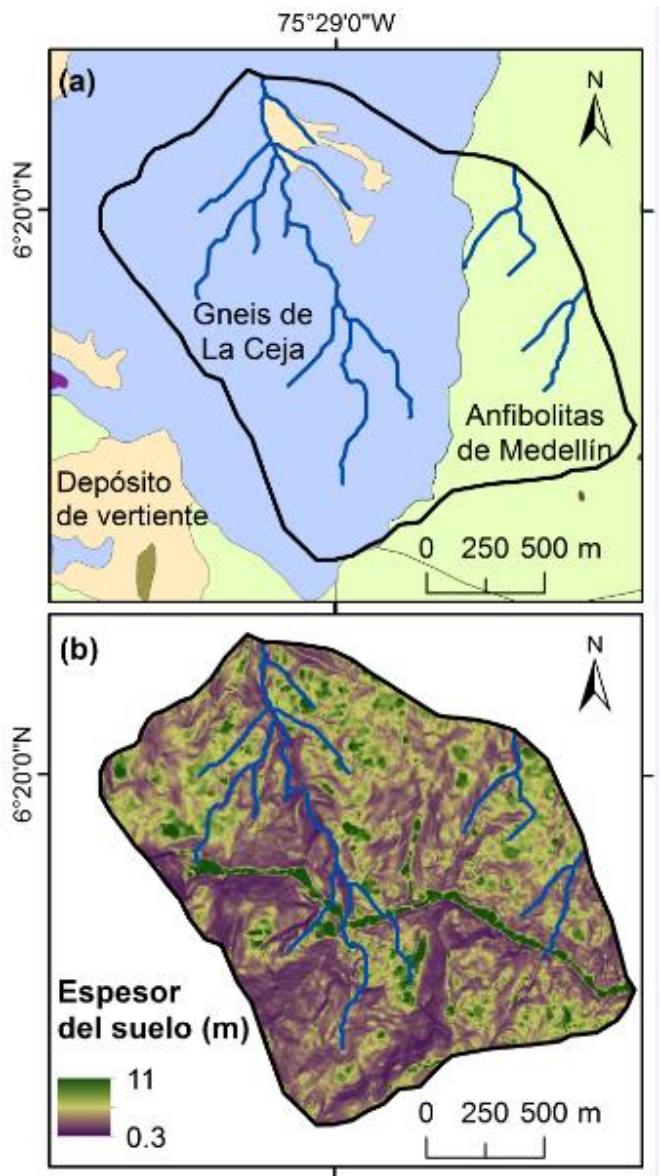

$75^{\circ} 29^{\prime} 0^{\prime \prime} W$

Fig. 2. Mapas de las condiciones del terreno: (a) Unidades geológicasgeotécnicas del suelo; (b) Espesor del suelo.

varía la presencia de limos, arcillas y suelos limo arcillosos, con espesores de hasta $4 \mathrm{~m}$. El Gneis de La Ceja está compuesto por cuarzo, feldespato y biotita, con un suelo residual de arena arcillosa de color rojizo. El horizonte superficial es un suelo homogéneo limo arenoso, con espesores de hasta $2 \mathrm{~m} \mathrm{[12].} \mathrm{La}$ Fig. $2 \mathrm{~b}$ presenta el mapa de espesor del suelo, calculado a partir 
de un modelo [13] que lo relaciona con la pendiente y ha sido ampliamente utilizado alrededor del mundo [14]-[16].

\section{B. Riesgo}

El riesgo de deslizamientos superficiales $(R)$ representa el conjunto de pérdidas de vidas humanas, heridos, daños materiales o pérdidas asociadas a las actividades económicas como resultado de la ocurrencia de este fenómeno (amenaza) y su efecto en elementos expuestos de acuerdo a su nivel de vulnerabilidad [8].

$$
R=P[T] * P[C \mid T] * u(C)
$$

Donde $R$ es el riesgo, $P[T]$ es la amenaza (probabilidad de ocurrencia de deslizamientos superficiales), $P[C \mid T]$ es la vulnerabilidad (probabilidad condicional de pérdidas dada la ocurrencia del deslizamiento) y $u(C)$ es el costo de las consecuencias.

\section{Amenaza}

La amenaza de deslizamientos superficiales es la probabilidad de ocurrencia de este fenómeno en un área definida y en un periodo de tiempo dado [17]. En esta investigación, la amenaza a deslizamientos superficiales detonados por lluvia se evalúa mediante un modelo físico distribuido simple: SHALSTAB [18], ampliamente utilizado y reconocido alrededor del mundo [19]-[22]. Este modelo presenta una simplificación de los procesos hidrológicos en estado estacionario para un análisis de estabilidad de talud infinito.

Basado en el modelo hidrológico TOPOG [23], se asume que el flujo se infiltra en un estrato de menor conductividad hidráulica y sigue rutas de flujo determinadas topográficamente [24]. Se desarrolla un patrón de humedad local $(W)$ en el suelo basado en el área contribuyente $(a)$, la pendiente $(\theta)$ y la transmisividad saturada $(T)$ de cada elemento topográfico (o celda, en este caso):

$$
W=\frac{Q a}{b T \operatorname{sen} \theta}
$$

donde $Q$ es la lluvia en estado estacionario y $b$ es la longitud de la celda (representando la unidad de área longitudinal del contorno en el que incide el área contribuyente). La transmisividad saturada representa el producto entre la conductividad hidráulica saturada $\left(K_{s}\right)$ y el espesor del suelo $(z)$. Asumiendo que la conductividad hidráulica saturada no varía con la profundidad, $W=h / z$, donde $h$ es el espesor del suelo saturado sobre la superficie deslizante (asumida como la base del estrato superficial).

El componente geotécnico se basa en un análisis de equilibrio límite de talud infinito empleando el criterio de falla de Mohr-Coulomb. Combinado con el modelo hidrológico, se deriva la ecuación que representa el criterio de falla, en términos del área contribuyente por unidad de área longitudinal, como se muestra en (3).

$$
\frac{a}{b} \geq \frac{T}{Q} \operatorname{sen} \theta\left[\frac{\rho_{s}}{\rho_{w}}\left(1-\frac{\tan \theta}{\tan \phi}\right)+\frac{c^{\prime}}{\rho_{w} g z \cos ^{2} \theta \tan \phi}\right],
$$

donde $\rho_{\mathrm{s}}$ es la densidad unitaria del suelo, $\rho_{\mathrm{w}}$ es la densidad unitaria del agua, $g$ es la aceleración gravitacional, $c$ ' la cohesión efectiva del suelo y $\phi$ el ángulo de fricción del suelo. Cuando se cumple la desigualdad de (3), la celda se predice como inestable; de lo contrario, las celdas son estables, ya que no tienen suficiente área contribuyente (y humedad local) para fallar. Las celdas incondicionalmente estables son aquellas que no predicen falla ni siquiera cuando están saturadas [18].

\section{Propiedades mecánicas e hidráulicas del suelo}

El modelo SHALSTAB requiere como insumos para el análisis de estabilidad un mapa de pendientes, mapa del espesor del suelo, propiedades mecánicas (cohesión, ángulo de fricción y densidad unitaria del suelo) e hidráulicas del suelo (conductividad hidráulica) e información de una lluvia constante. La Tabla 1 muestra los valores de los parámetros mecánicos e hidráulicos, asumidos constantes en toda el área de estudio.

\section{E. Probabilidad de excedencia de evento de lluvia detonante}

Se realiza un análisis estadístico para determinar la probabilidad de excedencia de diferentes acumulados de precipitación diaria en una estación pluviométrica del IDEAM (Instituto de Hidrología, Meteorología y Estudios Ambientales) denominada "La Cuchilla", ubicada en el Municipio de Girardota. Se cuentan con datos históricos de precipitación de los últimos 20 años. Su precipitación promedio mensual multianual, para el mes más lluvioso (octubre), es de $269 \mathrm{~mm}$. Se calcula la probabilidad de excedencia diaria de acumulados de lluvia cada $5 \mathrm{~mm}$, hasta $50 \mathrm{~mm}$, y se realiza un ajuste exponencial para determinar el acumulado de lluvia de una probabilidad de excedencia anual de $2 \%$ (Fig. 3). Se obtiene un acumulado de $50.0 \mathrm{~mm}$, de modo que la simulación con el

TABLA I

PROPIEDADES MECÁNICAS E HIDRÁULICAS DEL SUELO

\begin{tabular}{clc}
\hline \hline \multirow{2}{*}{ Símbolo } & \multicolumn{1}{c}{ Variable } & Valor \\
\hline$c^{\prime}$ & Cohesión $\left(\mathrm{kN} / \mathrm{m}^{2}\right)$ & 6 \\
$\phi$ & Angulo de fricción $\left(^{\circ}\right)$ & 36 \\
$\rho_{\mathrm{S}}$ & Densidad unitaria del suelo $\left(\mathrm{kg} / \mathrm{m}^{3}\right)$ & 1.69 \\
$K_{s}$ & Conductividad hidráulica saturada $(\mathrm{m} / \mathrm{s})$ & $1 * 10^{-7}$ \\
\hline \hline
\end{tabular}

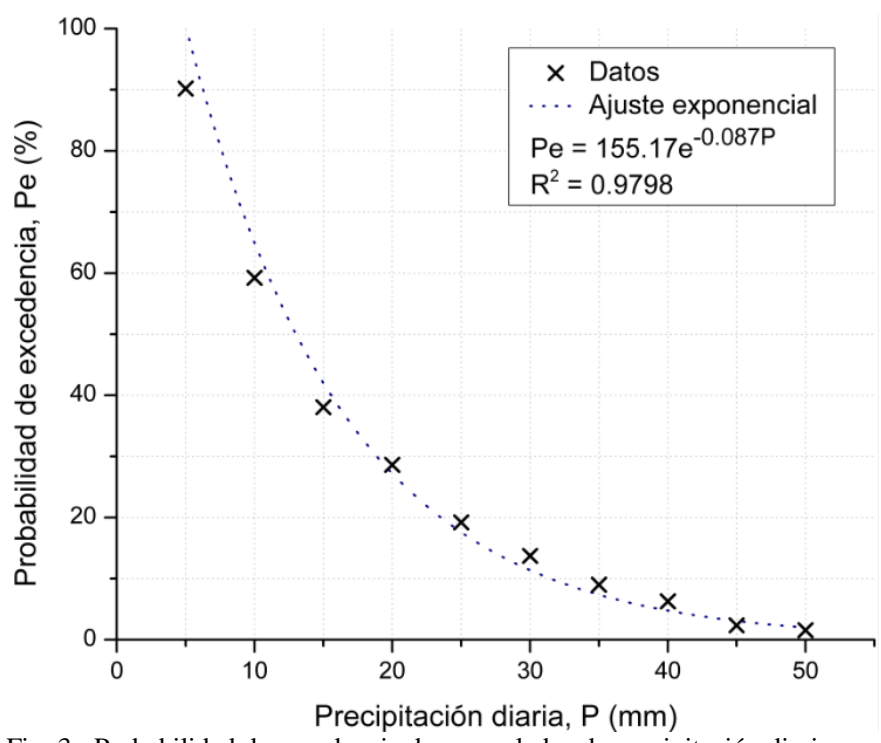

Fig. 3. Probabilidad de excedencia de acumulados de precipitación diaria con datos históricos de estación pluviométrica cercana al sitio de estudio. 
modelo SHALSTAB se realiza con una lluvia estacionaria de $50 \mathrm{~mm} /$ día.

\section{F. Vulnerabilidad}

Representa las pérdidas o daño de uno o varios elementos expuestos dentro del área de influencia de la amenaza (deslizamientos superficiales) [25]. Se emplea un modelo matemático que define el grado de pérdida esperado de una estructura en una escala de 0 (sin ningún daño) hasta 1 (destrucción total), denominado modelo $\mathrm{T}$ [26]. La vulnerabilidad física $(\mathrm{V})$ se determina mediante unos factores de intensidad y resistencia de la estructura, de acuerdo a (5) y (6).

$$
\begin{gathered}
V=\left[1+\left(\frac{T}{0.55}\right)^{-3.5}\right]^{-1}, \\
T=\frac{Z}{R_{s t r}},
\end{gathered}
$$

donde $T$ es la relación entre la resistencia de la estructura $\left(R_{s t r}\right)$ y el gradiente modificado $(Z)$, que relaciona la energía potencial y cinética que intervienen en la ocurrencia de este fenómeno:

$$
Z=\frac{H}{d}
$$

donde $H$ es la altura entre la cota máxima del deslizamiento y el elemento expuesto y $d$ la distancia a lo largo del terreno desde la cota máxima del deslizamiento hasta el centro de gravedad de la estructura [27].

La resistencia de la estructura $\left(R_{s t r}\right)$ se estima como [28]:

$$
R_{\text {str }}=\left(\varepsilon_{\text {sfd }} * \varepsilon_{\text {sty }} * \varepsilon_{\text {smn }} * \varepsilon_{\text {sht }}\right)^{0.25} \text {, }
$$

donde $\varepsilon_{\text {sty }}$ un factor del material de construcción de la estructura portante, $\varepsilon_{\mathrm{smn}}$ un factor del estado de mantenimiento y $\varepsilon_{\text {sht }}$ un factor de la altura (número de pisos). Los valores de estos factores se pueden determinar mediante las tablas propuestas por [28] (para $\varepsilon_{\text {sty }}, \varepsilon_{\text {smn }} \mathrm{y} \varepsilon_{\text {sht }}$ ), junto con una ecuación que relaciona la profundidad de la fundación $\left(D_{f o d}\right)$ con el factor $\varepsilon_{\text {sfd, }}$ como se muestra en (9):

$$
\varepsilon_{s f d}=\left(\frac{D_{f o d}}{10}\right)^{\frac{1}{3}}-0.05 \text {. }
$$

Li et al. (2010) [28] involucran el riesgo de una persona relacionando su resistencia con la habilidad de resistencia y evacuación al ser sorprendida por un deslizamiento de una intensidad dada. [29] analizaron la vulnerabilidad a movimientos en masa de personas en estructuras, así como a otras amenazas naturales y tecnológicas. Para la amenaza de deslizamientos se considera que la proporción entre lesiones leves, graves y fatalidades depende principalmente de la velocidad del movimiento. Se estima la probabilidad de una persona dentro de una estructura de sufrir lesiones de diferentes grados según la vulnerabilidad de la estructura. Li et al. (2010) [28] analizan los resultados de dicha investigación, recomendando una vulnerabilidad de 0.2 para lesiones leves, 0.8 para lesiones graves y 1.0 para fatalidades, y estiman la vulnerabilidad promedio de personas en estructuras mediante una función exponencial que ajusta la vulnerabilidad de personas dentro de una estructura $\left(V_{p-s}\right)$ con la vulnerabilidad de la estructura $\left(V_{s t r}\right)$, de acuerdo a (10):

$$
V_{p-s}=0.0014 * e^{6.07 V_{s t r}}
$$

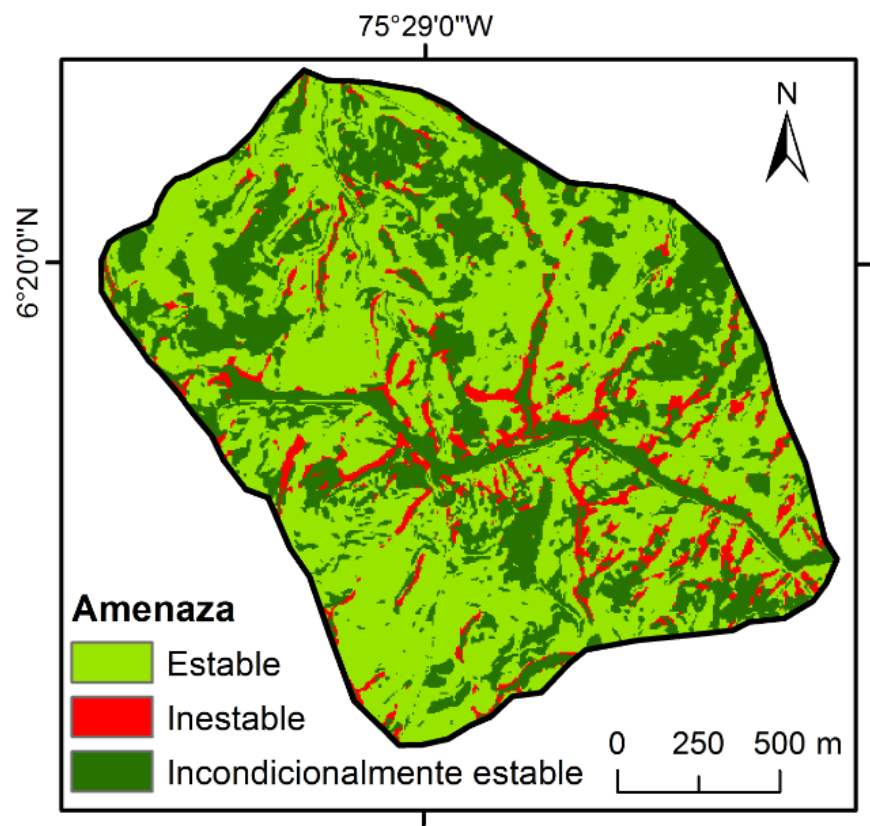

Fig. 4. Mapa de amenaza de deslizamientos superficiales para evento de lluvia con probabilidad de ocurrencia anual de $2 \%$.

\section{RESUltados}

La Fig. 4 muestra el mapa de amenaza de deslizamientos superficiales en el sitio de estudio, asociado a una probabilidad de excedencia anual de $2 \%$. En otras palabras, existe una probabilidad del $2 \%$ de ocurrencia anual (o excedencia) del evento desencadenante, un acumulado de lluvia de $50 \mathrm{~mm} /$ día, que causaría deslizamientos superficiales en las zonas que se identificaron como inestables.

Muchos modelos físicos distribuidos se han implementado en investigaciones en diferentes partes del mundo para analizar la ocurrencia de deslizamientos [30]-[35]. SHALSTAB, además de ser uno de los más simples en el planteamiento de las ecuaciones e implementación, por su sencilla (relativamente) utilización por medio de las herramientas de sistemas de información geográfica (SIG), se diferencia en la forma en que representa los resultados de estabilidad. La mayoría de modelos físicos distribuidos lo presentan en términos del factor de seguridad, que puede dar una noción cuantitativa de la susceptibilidad en una celda. En cambio, este modelo únicamente indica la condición de estabilidad o inestabilidad, aunque denote de otra manera las celdas que se predicen que fallan en cualquier condición de saturación (incondicionalmente inestables) y las que no fallan incluso con el suelo completamente saturado (incondicionalmente estables).

De esta manera, esta descripción de la estabilidad puede ser incorporada en la evaluación de la amenaza relacionándola directamente con la probabilidad de excedencia anual del evento detonante. En esta investigación, esta probabilidad se selecciona a criterio de los autores, considerando un nivel de susceptibilidad bajo como algunos investigadores determinan para probabilidades de falla menores a 5\% [36], [37]. Cabe destacar que hay una gran diferencia entre la probabilidad de 
excedencia del evento de lluvia y una probabilidad de falla como la que se ha determinado en diferentes investigaciones [9], [36]-[38] de susceptibilidad o amenaza de deslizamientos. Esta última generalmente describe la probabilidad de ocurrencia (espacial) asociada a unas condiciones del terreno y del factor externo detonante, que puede incorporar la incertidumbre en el análisis mediante las distribuciones de probabilidad de las variables aleatorias que inciden en el análisis de estabilidad (por ejemplo propiedades mecánicas e hidráulicas del suelo). En cambio, la probabilidad de excedencia anual de una lluvia que actúa como factor detonante de deslizamientos no se interpretaría directamente como un umbral del nivel de su amenaza, pero se podría elaborar un mapa de amenaza relacionando diferentes probabilidades de excedencia con determinados niveles de amenaza (por ejemplo $1 \%, 5 \%$ y $10 \%$ para delimitar zonas de amenaza baja, media y alta, respectivamente).

Esta investigación no pretende realizar una zonificación de amenaza que permita definir cuantitativamente o cualitativamente niveles de amenaza (baja, media o alta) en cada celda del sitio de estudio. De esta manera, es suficiente con la probabilidad de excedencia anual (2\%) seleccionada para determinar las condiciones de estabilidad o inestabilidad a lo largo del terreno.

\section{A. Viviendas analizadas}

Se analizan 8 viviendas ubicadas dentro del área de estudio a lo largo del tramo de la Autopista Medellín-Bogotá. Su ubicación se presenta en la Fig. 5, que ilustra los resultados de la evaluación de la vulnerabilidad física de las mismas definiendo tres clases: baja $(<0.4)$, media $(0.4-0.7)$ y alta $(>$ $0.7)$. Esta vulnerabilidad, como una probabilidad de pérdida condicionada a la ocurrencia del deslizamiento, fue obtenida a partir de los factores $\left(\varepsilon_{\mathrm{sfd}}, \varepsilon_{\mathrm{sty}}, \varepsilon_{\mathrm{smn}}\right.$ y $\left.\varepsilon_{\mathrm{sht}}\right)$ para la estimación de la resistencia de la estructura $\left(R_{\text {str }}\right)$, definidos para cada vivienda (según propuesta de $\mathrm{Li}$ et al., 2010 [28]), y el cálculo del gradiente modificado $(Z)$ de una celda, cercana y en una cota superior, en la que se predice inestabilidad en la evaluación de

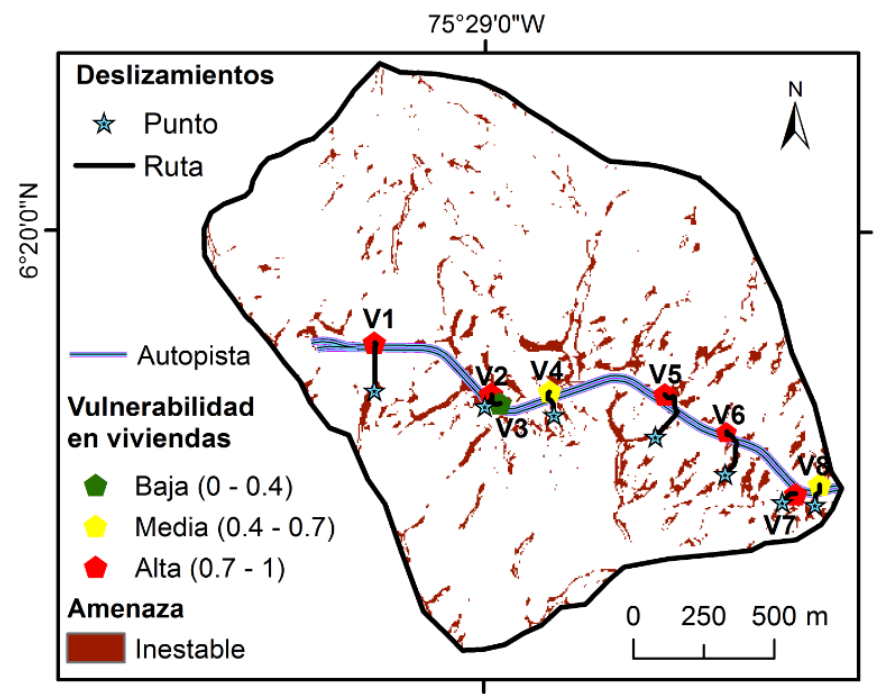

Fig. 5. Vulnerabilidad de 8 viviendas aledañas a la autopista Medellín-Bogotá dentro del sitio de estudio. la amenaza (Fig. 4). De esta forma, se asume que un deslizamiento en dicha celda afectaría la vivienda estudiada. Los parámetros requeridos en el análisis de la vulnerabilidad se presentan en la Tabla 2.

La Tabla 3 presenta la vulnerabilidad física $\left(V_{s t r}\right)$ de las 8 viviendas analizadas, su exposición o costo de las consecuencias $u(C)$ y el riesgo de deslizamientos superficiales $(R)$ para cada una de ellas. La exposición y el riesgo se calculan en términos económicos (dólar estadounidense). El costo de las consecuencias se obtuvo a partir de un estimativo del valor económico del metro cuadrado construido en el municipio de Copacabana \$76.47 (USD), según el catastro departamental. Finalmente, el riesgo de deslizamientos se calcula de acuerdo a (1), asumiendo el mismo valor de amenaza (0.2) para todas las viviendas.

Las viviendas V2 y V6 presentaron el mayor riesgo anual de deslizamientos superficiales (\$565 USD y 513\$ USD, respectivamente). En ambas viviendas se obtuvo el menor valor de resistencia de la estructura $R_{s t r}(0.40)$, influenciado por un bajo factor de altura $\left(\varepsilon_{\text {sht }}\right)$, al ser de un solo piso, y del factor relacionado con la profundidad de la cimentación $\left(\varepsilon_{\mathrm{sfd}}\right)$, calculado mediante (9). En ambas viviendas se asumió una profundidad de fundación $\left(D_{f o d}\right)$ de $0.5 \mathrm{~m}$. Excepto por la vivienda V7 (también con $D_{\text {fod }}=0.5 \mathrm{~m}$ ), en las demás se adoptó una profundidad de cimentación de $1.0 \mathrm{~m}$.

La vivienda V2 tiene el mayor grado de exposición en términos económicos (costo de pérdida) debido a que se asume proporcional a la valoración del metro cuadrado construido. De esta manera, incluso teniendo otras viviendas con mayor vulnerabilidad $(\mathrm{V} 1=0.98, \mathrm{~V} 5=0.85, \mathrm{~V} 6=0.87$ y V7 $=0.99)$, la estimación del riesgo anual es mayor al tener expuesta una mayor área. En contraste, la vivienda V3, siendo la segunda de mayor área $\left(498 \mathrm{~m}^{2}\right)$, tiene el menor riesgo anual (\$137 USD)

TABLA II

Variables Para El Cálculo De La Vulnerabilidad Física De Las VIVIENDAS

\begin{tabular}{cccccccc}
\hline \hline Vivienda & $\varepsilon_{\text {sfd }}$ & $\varepsilon_{\text {sty }}$ & $\varepsilon_{\text {smn }}$ & $\varepsilon_{\text {sht }}$ & $R_{\text {str }}$ & \multirow{2}{*}{$Z$} & $T$ \\
\hline V1 & 0.51 & 0.80 & 0.40 & 0.40 & 0.51 & 0.84 & 1.65 \\
V2 & 0.42 & 0.80 & 0.80 & 0.10 & 0.40 & 0.30 & 0.73 \\
V3 & 0.51 & 0.80 & 1.20 & 0.40 & 0.67 & 0.24 & 0.36 \\
V4 & 0.51 & 0.80 & 1.20 & 0.40 & 0.67 & 0.36 & 0.54 \\
V5 & 0.51 & 0.80 & 0.40 & 0.40 & 0.51 & 0.45 & 0.90 \\
V6 & 0.42 & 0.80 & 0.80 & 0.10 & 0.40 & 0.38 & 0.95 \\
V7 & 0.42 & 0.80 & 0.10 & 0.90 & 0.42 & 0.82 & 1.96 \\
V8 & 0.51 & 0.80 & 0.80 & 0.40 & 0.60 & 0.39 & 0.65 \\
\hline \hline
\end{tabular}

TABLA III

RIESGO DE LAS ESTRUCTURAS

\begin{tabular}{cccc}
\hline \hline Vivienda & $V_{\text {STR }}$ & $u(C)$ & $R$ (estructuras) \\
\hline V1 & 0.98 & $\$ 21,950$ & $\$ 430$ \\
V2 & 0.73 & $\$ 38,623$ & $\$ 565$ \\
V3 & 0.18 & $\$ 38,088$ & $\$ 137$ \\
V4 & 0.49 & $\$ 16,367$ & $\$ 160$ \\
V5 & 0.85 & $\$ 124,129$ & $\$ 2,101$ \\
V6 & 0.87 & $\$ 29,445$ & $\$ 513$ \\
V7 & 0.99 & $\$ 15,985$ & $\$ 316$ \\
V8 & 0.65 & $\$ 29,139$ & $\$ 377$ \\
\hline \hline
\end{tabular}


por su baja vulnerabilidad. Esto se debe principalmente al valor bajo del gradiente modificado $(Z=0.24)$ y la alta resistencia de la estructura $\left(R_{s t r}=0.67\right)$. A su vez, estos se asocian a la larga distancia entre la vivienda y el deslizamiento $(d=217.4 \mathrm{~m})$ y el buen estado de mantenimiento de la vivienda (al igual que los otros factores de resistencia), respectivamente.

La Tabla 4 muestra el riesgo anual por pérdidas de vidas humanas $(R)$, de acuerdo a (1). La vulnerabilidad de las personas en las estructuras $\left(V_{p-s}\right)$ se calcula con (10) como una función de la vulnerabilidad física de la estructura $\left(V_{s t r}\right)$. El costo de la exposición $u(C)$ se presenta en términos del número de vidas humanas. Este se calcula para cada vivienda de acuerdo a su área cuadrada, asumiendo que se tiene un habitante cada $25 \mathrm{~m}^{2}$, por cada piso. El valor obtenido se redondea a un número entero de personas expuestas en cada edificación. De esta manera, el riesgo anual de vidas humanas por deslizamientos se expresa en términos de cantidad de personas.

A nivel global, es común representar el riesgo en una gráfica que ilustra la probabilidad de excedencia de una amenaza y sus consecuencias, mediante las curvas F-N, denotadas así porque relacionan las frecuencias y el número de vidas perdidas (u otras consecuencias como costos económicos). Estas curvas sirven para comparar el riesgo con otras alternativas de diseño [39].

La Fig. 6 muestra la propuesta de [40] de un gráfico F-N con los riesgos anuales promedio asociados a diferentes instalaciones civiles tradicionales $\mathrm{y}$ otras estructuras $\mathrm{o}$ proyectos de gran tamaño. En el eje vertical se tienen las probabilidades anuales de ocurrencia y en el eje horizontal las consecuencias correspondientes a su falla. La líneas envolventes marcadas como "aceptable" y "marginalmente aceptable" reflejan riesgos inferidos por otras obras civiles [41]. En esta figura se presentan únicamente las ubicaciones de los puntos asociados a pérdidas de vidas humanas dentro de las estructuras, para 6 de las viviendas.

El costo monetario de las viviendas estudiadas se encuentra en el orden de $\$ 10^{4}$ (USD), por lo cual no se esperan consecuencias económicas de falla comparables con los otros tipos de proyectos. En la Fig. 6 no se incluyen los puntos asociados a las pérdidas económicas porque el riesgo de todas las viviendas es inferior al mínimo del gráfico F-N (\$10 4 USD) propuesto por Whitman (1984) [40]. Este riesgo anual de las estructuras (en pérdidas económicas) se encuentra en un orden de $\$ 10^{2}$ (USD), por lo cual para una probabilidad de falla anual de $10^{-2}$ se encontraría dentro de la envolvente de riesgo aceptable.

TABLA IV

RIESGO DE PERSONAS DENTRO DE LAS ESTRUCTURAS

\begin{tabular}{cccc}
\hline \hline Vivienda & $V_{P-S}$ & $u(C)$ & $R$ (personas) \\
\hline V1 & 0.53 & 11 & $1.2^{*} 10^{-1}$ \\
V2 & 0.12 & 20 & $4.8^{*} 10^{-2}$ \\
V3 & 0.00 & 20 & $1.7 * 10^{-3}$ \\
V4 & 0.03 & 9 & $4.9^{*} 10^{-3}$ \\
V5 & 0.24 & 6 & $2.9 * 10^{-2}$ \\
V6 & 0.28 & 15 & $8.3^{*} 10^{-2}$ \\
V7 & 0.56 & 8 & $9.0^{*} 10^{-2}$ \\
V8 & 0.07 & 15 & $2.1 * 10^{-2}$ \\
\hline \hline
\end{tabular}

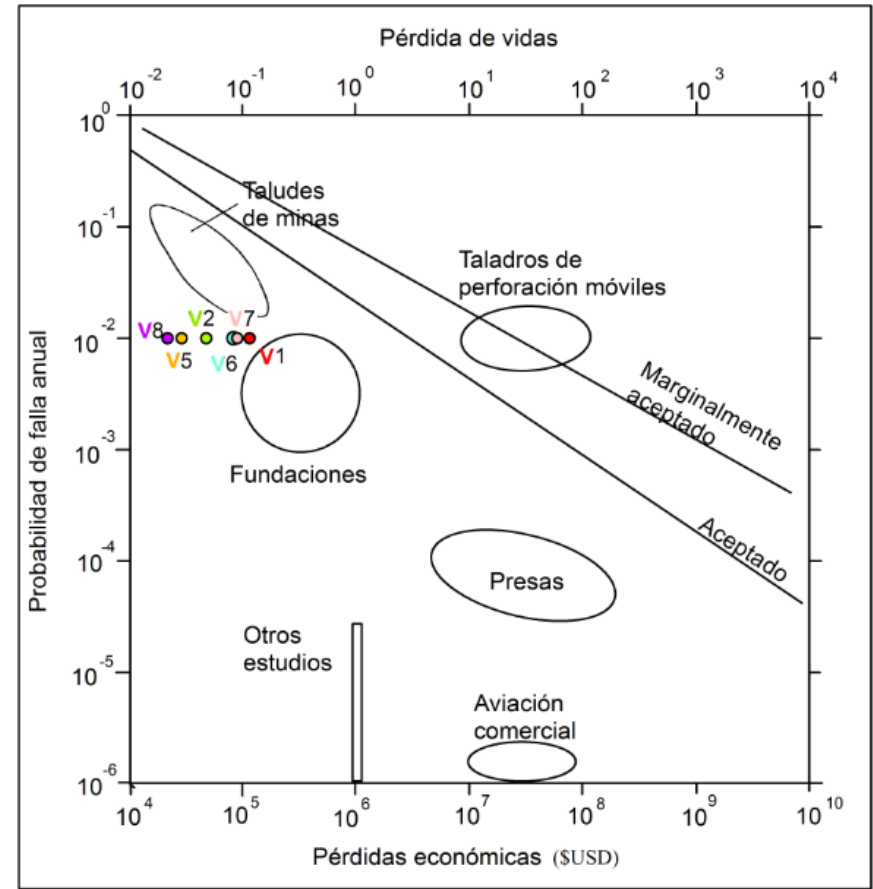

Fig. 6. Gráfico F-N con los riesgos anuales de pérdidas de vidas humanas para personas en las estructuras analizadas, comparado con los riesgos anuales promedio de diferentes instalaciones civiles tradicionales, estructuras o proyectos de gran tamaño. Adaptado de [40].

Por su parte, el riesgo de pérdidas humanas también entra dentro del riesgo aceptable para todas las viviendas, como se observa en los puntos representados en la Fig. 6. En este caso, se presentan diferencias significativas en los órdenes de los riesgos para las diferentes viviendas (entre $10^{-3}$ y $10^{-1}$ ), con el mayor valor para la vivienda V1. Nuevamente, dos de las viviendas (V3 y V4) tienen un riesgo inferior al valor mínimo representado en el gráfico F-N y sus puntos no se incluyen en la figura.

Las tasas de falla anual promedio de diferentes estructuras o proyectos, que se comparan con el riesgo de las viviendas analizadas en la Fig. 6, no constituyen un indicativo de riesgo aceptable cada caso. Sin embargo, el cálculo del riesgo para un problema ingenieril como el de las estructuras estudiadas sirve como una herramienta de gran utilidad en la comunicación entre los ingenieros, clientes y/o entidades reguladoras, para comparar entre posibles alternativas de solución (por ejemplo, previamente a la realización de un proyecto de infraestructura) o evaluar la forma en que se puede reducir el riesgo (por ejemplo, obras para mitigar la amenaza) [41].

\section{CONCLUSIONES}

Se implementó una metodología para analizar y evaluar la amenaza, vulnerabilidad y riesgo asociados a deslizamientos superficiales en un terreno montañoso tropical. Para la amenaza, se utilizó un modelo físico distribuido (SHALSTAB) ampliamente conocido en el mundo basado en una descripción fácil de implementar con las herramientas de un sistema de información geográfica y conceptualmente simple. De igual forma, se emplearon otros modelos para calcular la vulnerabilidad física (modelo T [26]) de una estructura y de las 
personas dentro de las mismas (Li et al., 2010 [28]). Los riesgos en términos económicos (para 8 viviendas) y de pérdidas de vidas humanas (para personas dentro de las viviendas) se calcularon mediante un análisis de las consecuencias de estos elementos expuestos dada la ocurrencia de deslizamientos superficiales definidos en la evaluación de la amenaza.

Una evaluación detallada de la amenaza de movimientos en masa podría incluir la descripción de su ubicación espacial, área o volumen, clasificación, velocidad del material movilizado y su ocurrencia dentro de un periodo de tiempo dado [1]. En esta investigación se implementó una metodología que se centra en determinar la localización de los posibles movimientos y se relaciona con un evento de lluvia en estado estacionario obtenido a partir de una probabilidad de excedencia anual que fue seleccionada para llevar a cabo el análisis. Si bien es un método simple, se puede extender a un análisis en el cual se pretenda zonificar para determinar zonas de amenaza baja, media y alta.

Los modelos para estimar la vulnerabilidad de una estructura y/o personas dentro de las mismas se basan en parámetros cuyo cálculo requiere mayor calibración e investigación. Si bien los autores presentan diferentes valores para los parámetros de resistencia según las condiciones de la estructura, tienen que ser interpretados como propuestas iniciales y su implementación debe conllevar un análisis crítico profundo [28]. Sin embargo, la posibilidad de cuantificar la vulnerabilidad es un gran aporte para la evaluación del riesgo de movimientos en masa.

La metodología que se implementó para analizar y evaluar el riesgo de deslizamientos superficiales representa una herramienta que puede ser útil en planes de ordenamiento territorial y gestión del riesgo de movimientos en masa. Su simplicidad y fácil aplicación, comparado con otros modelos físicos [42], [43] o metodologías para análisis del riesgo [8], [9], [16] que han sido implementadas en esta región, constituye una buena alternativa para muchos proyectos de infraestructura.

\section{REFERENCES}

R. Fell, J. Corominas, C. Bonnard, L. Cascini, E. Leroi, and W. Z. Savage, "Guidelines for landslide susceptibility, hazard and risk zoning for land-use planning," Eng. Geol., vol. 102, no. 3, pp. 99111, 2008. DOI: 10.1016/j.enggeo.2008.03.014

[2] R. Talaei, "Landslide risk assessment using a multi-method approach in Hashtchin region (NW of Iran)," Acta Geod. Geophys., vol. 49, no. 3, pp. 381-401, 2014. DOI: 10.1007/s40328-014-0052-x

[3] L. Vranken, G. Vantilt, M. Van Den Eeckhaut, L. Vandekerckhove, and J. Poesen, "Landslide risk assessment in a densely populated hilly area," Landslides, vol. 12, no. 4, pp. 787-798, 2015. DOI: 10.1007/s10346-014-0506-9

[4] M. Bednarik, I. Yilmaz, and M. Marschalko, "Landslide hazard and risk assessment: a case study from the Hlohovec-Sered'landslide area in south-west Slovakia," Nat. hazards, vol. 64, no. 1, pp. 547-575, 2012. DOI: 10.1007/s11069-012-0257-7.

[5] B. Huang, Y. Yin, and J. Tan, "Risk assessment for landslide-induced impulse waves in the Three Gorges Reservoir, China," Landslides, vol. 16, no. 3, pp. 585-596, 2019. DOI: 10.1007/s10346-018-1115-9

[6] J. Corominas et al., "Recommendations for the quantitative analysis of landslide risk," Bull. Eng. Geol. Environ., vol. 73, no. 2, pp. 209263, 2014. DOI: $10.1007 / \mathrm{s} 10064-013-0538-8$

[7] L. Cascini, S. Ferlisi, and E. Vitolo, "Individual and societal risk owing to landslides in the Campania region (southern Italy)," Georisk, vol. 2, no. 3, pp. 125-140, 2008. DOI: $10.1080 / 17499510802291310$

[8] J. A. Vega and C. A. Hidalgo, "Quantitative risk assessment of landslides triggered by earthquakes and rainfall based on direct costs of urban buildings," Geomorphology, vol. 273, pp. 217-235, 2016. DOI: 10.1016/j.geomorph.2016.07.032

[9] R. J. Marín, J. C. Guzmán-Martínez, H. E. Martínez, E. F. GarcíaAristizábal, J. D. Cadavid-Arango, and P. Agudelo-Vallejo, "Evaluación del riesgo de deslizamientos superficiales para proyectos de infraestructura: caso de análisis en vereda El Cabuyal," Ing. y Ciencia, vol. 14, no. 27, pp. 153-177, 2018. DOI: 10.17230/ingciencia.14.27.7

[10] P. A. Isaza-Restrepo, H. E. Martínez, and C. A. Hidalgo, "Methodology for quantitative landslide risk analysis in residential projects," Habitat Int., vol. 53, pp. 403-412, 2016. DOI: 10.1016/j.habitatint.2015.12.012

[11] E. Aristizábal and J. Gómez, "Inventario de emergencias y desastres en el Valle de Aburrá. Originados por fenómenos naturales y antrópicos en el período 1880-2007," Gestión y Ambient., vol. 10, no. 2, pp. 17-30, 2007.

[12] AMVA and Universidad Nacional de Colombia-UNAL, Estudios básicos de amenaza por movimientos en masa, inundaciones y avenidas torrenciales en los municipios de Caldas, La Estrella, Envigado, Itagüí, Bello, Copacabana y Barbosa, para la incorporación de la gestión del riesgo en la planificación territori. Medellín: Área Metropolitana del Valle del Aburrá, 2018.

[13] G. M. Saulnier, K. Beven, and C. Obled, "Including spatially variable effective soil depths in TOPMODEL," J. Hydrol., vol. 202, no. 1-4, pp. $158-172,1997$. DOI: $10.1016 /$ S0022-1694(97)00059-0

[14] F. Catani, S. Segoni, and G. Falorni, "An empirical geomorphologybased approach to the spatial prediction of soil thickness at catchment scale," Water Resour. Res., vol. 46, no. 5, 2010. DOI: 10.1029/2008WR007450

[15] T. The Viet, M. Alvioli, G. Lee, and H. U. An, "Three-dimensional, time-dependent modeling of rainfall-induced landslides over a digital landscape: a case study," Landslides, vol. 15, no. 6, pp. 1071-1084, 2018. DOI: 10.1007/s10346-017-0931-7

[16] R. J. Marin and Á. J. Mattos, "Physically-based landslide susceptibility analysis using Monte Carlo simulation in a tropical mountain basin," Georisk Assess. Manag. Risk Eng. Syst. Geohazards, vol. 13, no. 3, pp. 1-14, 2019. DOI: 10.1080/17499518.2019.1633582

[17] P. Jaiswal, C. J. van Westen, and V. Jetten, "Quantitative landslide hazard assessment along a transportation corridor in southern India," Eng. Geol., vol. 116, no. 3, pp. 236-250, 2010. DOI: 10.1016/j.enggeo.2010.09.005

[18] D. R. Montgomery and W. E. Dietrich, "A physically based model for the topographic control on shallow landsliding," Water Resour. Res., vol. 30, no. 4, pp. 1153-1171, 1994. DOI: 10.1029/93WR02979 M. Teixeira, C. Bateira, F. Marques, and B. Vieira, "Physically based shallow translational landslide susceptibility analysis in Tibo catchment, NW of Portugal," Landslides, vol. 12, no. 3, pp. 455-468, 2015. DOI: 10.1007/s10346-014-0494-9

[20] M. S. Kim, Y. Onda, T. Uchida, and J. K. Kim, "Effects of soil depth and subsurface flow along the subsurface topography on shallow landslide predictions at the site of a small granitic hillslope," Geomorphology, vol. 271, pp. 40-54, 2016. DOI: 10.1016/j.geomorph.2016.07.031

[21] A. M. S. Pradhan and Y. T. Kim, "Evaluation of a combined spatial multi-criteria evaluation model and deterministic model for landslide susceptibility mapping," Catena, vol. 140, pp. 125-139, 2016. DOI: 10.1016/j.catena.2016.01.022

[22] R. M. Sbroglia, G. M. P. Reginatto, R. A. R. Higashi, and R. F. Guimarães, "Mapping susceptible landslide areas using geotechnical homogeneous zones with different DEM resolutions in Ribeirão Baú basin, Ilhota/SC/Brazil," Landslides, vol. 15, no. 10, pp. 2093-2106, 2018. DOI: 10.1007/s10346-018-1052-7

[23] E. M. O'Loughlin, "Prediction of surface saturation zones in natural catchments by topographic analysis," Water Resour. Res., vol. 22, no. 5, pp. 794-804, 1986. DOI: 10.1029/WR022i005p00794

[24] R. F. Guimaraes, D. R. Montgomery, H. M. Greenberg, N. F. Fernandes, R. A. T. Gomes, and O. A. de Carvalho Júnior, "Parameterization of soil properties for a model of topographic controls on shallow landsliding: application to Rio de Janeiro," Eng. Geol., vol. 69, no. 1-2, pp. 99-108, 2003. DOI: 10.1016/S00137952(02)00263-6

[25] R. Fell, K. K. S. Ho, S. Lacasse, and E. Leroi, "A framework for landslide risk assessment and management," Landslide risk Manag., 
pp. $163-202,2005$.

H. E. Martínez-Carvajal, M. T. de Moraes Guimarães Silva, E. F. García-Aristizábal, E. V Aristizábal-Giraldo, and M. A. LariosBenavides, "A mathematical approach for assessing landslide vulnerability," Earth Sci. Res. J., vol. 22, no. 4, pp. 251-273, 2018. DOI: $10.15446 /$ esrj.v22n4.68553

[27] M. T. de M. Guimarães Silva, "Avaliação quantitativa da vulnerabilidade física associada a processos de deslizamentos de encostas," Universidade de Brasília, 2016.

[28] Z. Li, F. Nadim, H. Huang, M. Uzielli, and S. Lacasse, "Quantitative vulnerability estimation for scenario-based landslide hazards," Landslides, vol. 7, no. 2, pp. 125-134, 2010. DOI: 10.1007/s10346009-0190-3

[29] A. L. Ragozin and I. O. Tikhvinsky, "Landslide hazard, vulnerability and risk assessment," in 8th international symposium on landslides Cardiff, 2000, pp. 1257-1262.

[30] R. T. Pack, D. G. Tarboton, and C. N. Goodwin, "The SINMAP approach to terrain stability mapping," in 8th congress of the international association of engineering geology, Vancouver, British Columbia, Canada, 1998, pp. 21-25.

[31] L. Montrasio and R. Valentino, "A model for triggering mechanisms of shallow landslides," Nat. Hazards Earth Syst. Sci., vol. 8, no. 5, pp. 1149-1159, 2008. DOI: 10.5194/nhess-8-1149-2008

[32] R. L. Baum, W. Z. Savage, and J. W. Godt, "TRIGRS- A Fortran Program for Transient Rainfall Infiltration and Grid-Based Regional Slope-Stability Analysis, Version 2. 0," U.S. Geological Survey Open-File Report, 2008.

[33] E. Aristizábal, J. I. Vélez, H. E. Martínez, and M. Jaboyedoff, "SHIA_Landslide: a distributed conceptual and physically based model to forecast the temporal and spatial occurrence of shallow landslides triggered by rainfall in tropical and mountainous basins," Landslides, vol. 13, no. 3, pp. 497-517, 2016. DOI: 10.1007/s10346015-0580-7

[34] M. E. Reid, S. B. Christian, D. L. Brien, and S. Henderson, "Scoops3D-Software to Analyze Three-Dimensional Slope Stability Throughout a Digital Landscape," 2015. DOI: $10.3133 / \mathrm{tm} 14 \mathrm{~A} 1$

[35] R. M. Iverson, "Landslide triggering by rain infiltration," Water Resour. Res., vol. 36, no. 7, p. 1897, 2000. DOI: 10.1029/2000WR900090

[36] J. H. Lee and H. J. Park, "Assessment of shallow landslide susceptibility using the transient infiltration flow model and GISbased probabilistic approach," Landslides, vol. 13, no. 5, pp. 885903, 2016. DOI: 10.1007/s10346-015-0646-6

[37] H. J. Park, J. Y. Jang, and J. H. Lee, “Assessment of rainfall-induced landslide susceptibility at the regional scale using a physically based model and fuzzy-based Monte Carlo simulation," Landslides, pp. 119, 2019. DOI: $10.1007 / \mathrm{s} 10346-018-01125-\mathrm{z}$

[38] C.-N. Liu and C. C. Wu, "Mapping susceptibility of rainfall-triggered shallow landslides using a probabilistic approach," Environ. Geol., vol. 55, no. 4, pp. 907-915, 2008. DOI: 10.1007/s00254-007-1042-x

[39] G. B. Baecher and J. T. Christian, Reliability and statistics in geotechnical engineering. London: John Wiley \& Sons Ltd, 2003.

[40] R. V Whitman, "Evaluating calculated risk in geotechnical engineering," J. Geotech. Eng., vol. 110, no. 2, pp. 143-188, 1984. DOI: 10.1061/(ASCE)0733-9410(1984)110:2(143)

[41] R. V Whitman, "Organizing and evaluating uncertainty in geotechnical engineering," J. Geotech. Geoenvironmental Eng., vol. 126, no. 7, pp. 583-593, 2000. DOI: 10.1061/(ASCE)10900241(2000)126:7(583)

[42] R. J. Marín, and M. F. Velásquez, "Influence of hydraulic properties on physically modelling slope stability and the definition of rainfall thresholds for shallow landslides," Geomorphology, vol. 351, no. 15, pp. 106976, 2020. DOI: 10.1016/j.geomorph.2019.106976

[43] R. J. Marín, E.F. García, and E. Aristizábal, "Umbrales de lluvia para deslizamientos superficiales basados en modelos físicos: aplicación en una subcuenca del Valle de Aburrá (Colombia)," DYNA, vol. 86, no. 210 , pp. 312-322, 2019. DOI: 10.15446/dyna.v86n210.77166
Roberto J. Marín nació en Medellín (Colombia) en 1991. Obtuvo sus títulos de Ingeniero Civil (2015) y Master en Ingeniería (2018) en la Universidad de Antioquia. Es investigador, docente de cátedra y estudiante del Doctorado en Ingeniería Ambiental en la Universidad de Antioquia. Las áreas de interés en investigación son: estabilidad de laderas, modelación de movimientos en masa, evaluación del riesgo geotécnico, umbrales de lluvia para deslizamientos, entre otras. ORCID: http://orcid.org/0000-0002-3206-0425

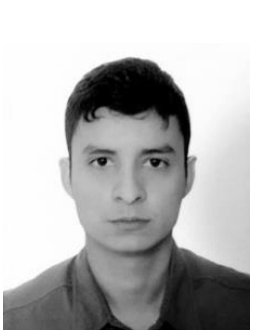

Jesner Marín-Londoño nació en Puerto Triunfo (Colombia) en 1996. Obtuvo su título de Ingeniero Civil (2019) en la Universidad de Antioquia. Es investigador de la Universidad de Antioquia y actualmente ingeniero residente de puentes para el proyecto Autopista Conexión Pacífico 1. Las áreas de interés en investigación son: Estabilidad de taludes, Movimientos en masa detonados por lluvia, umbrales de lluvia para deslizamientos, riesgo geotécnico, entre otras.

ORCID: http://orcid.org/0000-0001-5892-9945

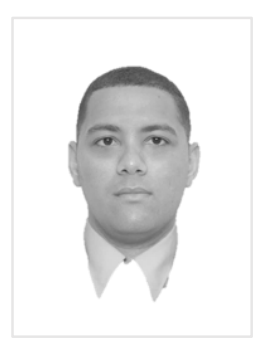

Álvaro J. Mattos nació en Villanueva (Colombia) en 1992. Obtuvo sus títulos de Ingeniero Civil (2015) y Máster en Ingeniería (2020) en la Universidad de Antioquia. Es investigador y docente en el Departamento de Ingeniería Sanitaria y Ambiental de la Universidad de Antioquia. $\mathrm{Su}$ campo de investigación incluye el modelado de la incertidumbre del suelo para la evaluación de riesgos de deslizamientos de tierra, análisis basados en la confiabilidad de estructuras geotécnicas, entre otros temas.

ORCID: http://orcid.org/0000-0003-1288-3288 